\title{
Et si la FMH déménageait de Berne à Zurich?
}

\section{Remo Osterwalder}

Dr méd., vice-président de la FMH, responsable du département Prestations et développement professionnel

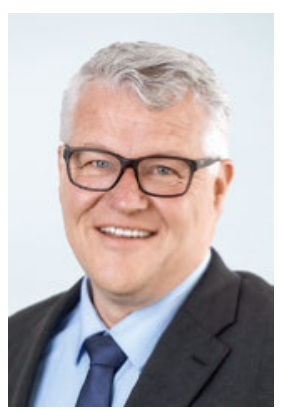

La question est certainement audacieuse mais l'impartialité liée à mes origines - j'ai grandi dans le NordOuest de la Suisse - me permet d'aborder la question sans idée préconçue.

Récemment de passage sur la Paradeplatz à Zurich, j'ai été impressionné par l'imposante force de l'art financier. Et nous voilà déjà au cœur du débat autour du corps médical: les médias et les politiciens parlent avant tout d'argent et de financement de la santé. Et qui sait mieux gérer l'argent que les spécialistes de la finance? 1:0 pour Zurich. Comme la question de la légalité d'une mesure médicale se pose toujours plus souvent dans notre système de santé humaniste, le corps médical est, à plusieurs titres, de plus en plus tributaire du soutien des juristes. Sur la liste des études d'avocats les plus prestigieuses de Suisse, les quatre premiers rangs sont occupés par celles dont le siège principal est à Zurich: $2: 0$ pour Zurich.

\section{Quand on est originaire du Nord-Ouest de la Suisse, on peut poser la question épineuse «Berne ou Zurich?».}

Le point suivant concerne le manque de place: la FMH a cruellement besoin de bureaux supplémentaires pour répondre au nombre croissant de postes de travail. Actuellement, seuls $63000 \mathrm{~m}^{2}$ de bureaux sont vides en ville de Berne alors qu'à Zurich, l'offre est nettement supérieure avec $398000 \mathrm{~m}^{2}$. Par conséquent, 3:0 pour Zurich. Lorsqu'on perd la vue d'ensemble, il est recommandé de prendre un peu de recul pour faire le tour de la situation. Personne ne niera que l'épicentre des décisions politiques se trouve à Berne. Comment réagir pour éviter le tsunami? S'éloigner des berges en fuyant vers le centre du pays. Pour cette raison, 4:0 pour Zurich. Penchons-nous à présent sur la théâtralité. Comme le dit le texte de la chanson de Queen «The Show Must Go
On»: «Another hero, another mindless crime» et «Does anybody want to take it anymore?», dans laquelle il est question de grand spectacle et de qui est le meilleur animateur. Aucun Bâlois ne me contredirait si j'affirmais que les Zurichois ont une plus grande "gueule» que les Bernois (excusez-moi, chers Zurichois, mais je ne pouvais pas me retenir de faire cette remarque). Mais c'est quand même un point pour vous. Zurich: 5:0. J'ai bien conscience que cette liste n'est pas exhaustive et qu'on pourrait y ajouter un bon nombre d'argu-

\section{Il peut être judicieux d'évaluer régulièrement} la situation et d'ajuster la direction à suivre.

ments. Pour moi, il s'agit avant tout de montrer qu'il peut être judicieux d'évaluer régulièrement la situation et d'ajuster la direction à suivre. Certes, de tels changements radicaux relèvent souvent plutôt du fantasme. Car, comment pourrait-on obliger une vieille dame de plus de nonante ans à suivre une cure de rajeunissement. Je vois déjà les courriers des lecteurs enflammés demandant comment peut-on oser remettre en question une culture développée pendant près d'un siècle. Il ne s'agit pas de réinventer la roue mais cette roue devrait au moins pouvoir tourner et les parties usagées être remplacées. Chers amis bernois, ne pensez pas que j'ai une dent contre vous. Bien au contraire, votre ville est formidable et pleine de charme dans une atmosphère sympathique. De plus, le fait que le chemin le plus direct pour se rendre à la salle des pas perdus est beaucoup plus court de Berne que depuis Zurich la rend encore plus attractive. N'ayez pas peur, le plus dangereux à Zurich reste l'énorme feu pour brûler le Böögg et la nostalgie de la cavalerie. Mais il n'en demeure pas moins, si vous me permettez de paraphraser Martin Luther King: I have a dream. 